\title{
Urinary Urgency Medications May Compromise Discrete rather than Global Cognitive Skills
}

\author{
Marilee Monnot Elliott Ross \\ Department of Neurology, University of Oklahoma Health Sciences Center and Veterans \\ Affairs Medical Center, Oklahoma City, Okla., USA
}

\section{Key Words}

Aging and cognition • Neuropsychology • Oxybutynin • RBANS • Reliable change index • Tolterodine $\cdot$ Urinary urgency

\begin{abstract}
Background/Aims: Prior research about cognitive problems associated with the use of urinary urgency medication (UUM) has reported mixed results that suggest procedures and/or assessments may need to be refined. Methods: Ten elderly subjects who were actively taking a UUM were assessed with neuropsychological testing before and after a 4-week UUM washout period. Results were evaluated by examining discrete subtest results, full-scale scores, and the reliable change index methodology. Results: Four controls and 5 subjects with mild cognitive impairment showed significant improvement in at least one subtest score on well-characterized instruments. Conclusion: In this case study of 10 subjects, withdrawal of oxybutynin and tolterodine resulted in significant changes in subtest scores with different patterns for each subject that were not necessarily reflected in their total scores. Thus, future clinical studies should always include analysis of subtest results as these changes may be the only indication that cognition has been improved or has declined significantly.

Copyright $\odot 2012$ S. Karger AG, Basel
\end{abstract}

\section{Introduction}

Cognitive Effects of Urinary Urgency Medications

It is well known that the elderly are sensitive to medications with anticholinergic side effects. The reason for this sensitivity may well relate to normal aging effects that cause a 
Monnot et al.: Urinary Urgency Medications May Compromise Discrete rather than Global Cognitive Skills

substantial loss of neurons in the nucleus basalis of Meynert, the sole source of cerebral acetylcholine innervation of muscarinic receptors [1]. Alzheimer's disease (AD) is associated with an accelerated loss of basalis neurons. Therefore, elderly patients, especially those with amnestic mild cognitive impairment (MCI) who have a high probability of converting to AD, may have excessive loss of basalis neurons, thus reducing their reserve capacity for cholinergic transmission. Many elderly patients have urge urinary incontinence and some have cognitive decline requiring treatment with cholinesterase inhibitor medication. In general, urinary urgency/incontinence medications (UUM) are anticholinergic. Tolterodine is an antimuscarinic UUM with low brain penetrance, and even though it is a tertiary amine, its distribution into the central nervous system is more similar to a quaternary amine anticholinergic drug. Nevertheless, in elderly subjects who may have reduced central cholinergic transmission, even small increases in antimuscarinic activity could lead to cognitive problems, including lethargy, alterations in attention, and memory loss [2, 3]. Oxybutynin chloride, another antimuscarinic UUM, is an antispasmodic agent that also crosses the bloodbrain barrier. It has been reported to be associated with delirium with associated EEG changes and alterations in rapid eye movement sleep activity [4-7]. Studies using oxybutynin have shown that increased serum anticholinergic levels are associated with decreased cognitive levels in demented patients $[8,9]$. A more recent study has shown that serum anticholinergic levels vary greatly among 107 drugs with anticholinergic effects [10]. In addition, concomitant use of anticholinergic and cholinesterase inhibitor medications in cognitively impaired patients has been associated with an increased use of atypical antipsychotic medications for the control of aberrant behavioral problems [11].

Conversely, one study has suggested that untreated urinary incontinence/urgency is associated with an increased risk for falls and non-spine non-traumatic fractures in older women, due to rushing to the toilet [12]. Other studies have shown no cognitive decline in (1) cognitively impaired female nursing home residents prescribed short-term low-dose oxybutynin, (2) nursing home residents, functioning at lower cognitive levels, who were taking both oxybutynin and a cholinesterase inhibitor for dementia, and (3) young children prescribed oxybutynin for urinary incontinence [13-15]. Randomized, double-blind studies of other UUMs such as solifenacin and darifenacin, with relatively low selectivity for the M1 muscarinic receptor, have shown no cognitive decline in healthy and impaired elderly subjects when directly compared to comparable doses of oxybutynin, which have been associated with cognitive problems $[16,17]$. Methodological differences between studies may have produced contradictory results that have failed to provide definitive information about the safety and effectiveness of UUMs in the elderly population.

\section{Reliable Change Index for Detection of Inter-Subtest Scores}

Studies using multifactorial neurobehavioral assessments must deal with variations in test score changes. Some subjects show increased test scores and some show decreases, so that group mean numbers may not describe the full extent of cognitive changes because the extremes balance out one another. In addition, practice effects, random measurement errors, and inter-subtest discrepancies may obscure the results of a study because a significant change in a subtest score may not be reflected in the group mean or total scores $[18,19]$. Recently, a strategy has been applied to detect significant changes in individual test scores so that medical interventions can be dependably assessed using the reliable change index (RCI) $[20,21]$. RCI calculations are relatively simple for a specific study group (see Statistical Methods); they produce upper and lower reliability figures for each test section/component with different rates of errors. Thus, RCI scores can be used to assess whether an individual subject changes significantly in each cognitive realm after a medical intervention. These RCI scores result in fewer type I and II errors when assessing subject test score changes. Thus, the direc- 
tion and magnitude of change in an individual cognitive test score can be viewed properly, without resorting to group means that may hide evidence of significant differences in discrete cognitive skills. Because this study focused on the methodology used to detect changes after medical intervention, the RCI technique was used to assess results.

The objective of this study was to determine if a UUM (oxybutynin or tolterodine) was associated with cognitive and behavioral decline in elderly patients diagnosed with MCI and in cognitively healthy elderly subjects. Mixed results shown in previously published studies about this subject may have been associated with the inclusion of subjects who were already demented; for such subjects, self-reported observations may be suspect, and their subtest scatter has been found to be as diminished as the total test score, due to various levels of incompetence [22]. The emphasis in the present study was on assessment instruments and methodology, using an extended washout period for the UUM, with family report diaries of each subject's behavior throughout the study. Inspection of cognitive subtest scores that record individual skills, rather than total instrument scores, was emphasized; the hypothesis to be tested was that the pattern of subtest scores would be significantly different for each individual after the washout period, but that total score changes would not necessarily show these significant differences. If this is true, it may explain some of the mixed results reported in prior studies and the complaints by patients using UUMs that their thinking processes and cognitive performance were impaired.

\section{Subjects and Methods}

Subjects for the study were recruited from the Center for Alzheimer's and Neurodegenerative Disorders, Veterans Affairs Medical Center (VAMC) and the OU Physicians outpatient clinics at the University of Oklahoma Health Sciences Center (OUHSC), Oklahoma City, Okla., USA. All read and signed both the VAMC Research and Development Committee and the OUHSC Institutional Review Board-approved informed consents prior to entrance into the study. All were taking either oxybutynin or tolterodine for urinary urgency for 6 months to 5 years prior to this study. Once enrolled, all subjects underwent cognitive testing prior to stopping their UUMs for 4 weeks and were re-tested at the end of the 4 -week washout period. Three categories of individuals were recruited: (1) subjects with normal cognitive functioning for their age and education levels, (2) subjects diagnosed with MCI, and (3) subjects diagnosed with MCI taking a cholinesterase inhibiter medication. Inclusion criteria were: (1) males and females between 60 and 85 years of age from any ethnic background, (2) use of a UUM for at least 6 months, and (3) proficient in the English language because the testing instruments and questionnaires were standardized for English speakers. Patients were excluded if they were taking medications that could distort cognitive test scores (e.g. antipsychotics, sedatives, narcotic analgesics, etc.), if they had chronic neurologic disorders (stroke, Parkinson's disease (PD), multiple sclerosis, traumatic brain injury, etc.), or if they had major chronic psychiatric disorders (schizophrenia, bipolar disease, depression).

Subjects were cognitively assessed before and after the washout period using the MiniMental State Examination (MMSE) [23, 24] and the Repeatable Battery for the Assessment of Neuropsychological Status (RBANS), alternating use of forms A and B [25]. The MMSE is a standard screening device allowing a brief bedside examination of orientation, memory, language, and visuospatial skills for any patient suspected of having cognitive changes (delirium, brain damage, dementia, etc.). The RBANS is a more extensive screening instrument that yields five index scores derived from eleven subtests of discrete cognitive skills. RBANS index scores include immediate memory, visuospatial construction, language, attention, and delayed memory. The test has been standardized for individuals spanning 20-89 years of age 
with index scores being expressed in percentiles, a standard metric by which individuals from different age groups can be compared and which can be readily understood by any clinical provider [25]. In addition, this instrument has been extensively assessed and used by neuropsychologists at the OUHSC [18].

Each subject's family member reported on the subject's behaviors using the Dementia Screening Questionnaire [26]. This clinical instrument includes the modified Clinical Dementia Rating scale (CDR; scoring of $0-3.5$ points for each of the eight categories, resulting in a total point count), Activities of Daily Living Scale (ADL), Instrumental Activities of Daily Living Scale (IADL), and the modified Frontal Behavioral Inventory (FBI). A diary noting 'urinary accidents per day' and 'cognitive problems per day' was filled out by the family member living with the subject, for the first week to establish baseline data while taking their prescribed UUM and during the subsequent 4 weeks of the UUM washout period. In addition, each subject filled out a Bladder Health Questionnaire at the beginning and end of the study to detect any changes in the subject's distress level due to the urinary accident rate before and after using a UUM. A medication dosing diary was kept for each patient. Before the study was started, criteria for significant changes in mental status were set at $\geq 3$ MMSE points and a change of $\geq 15$ points of the percentile rank of any index score or the total score for the RBANS. Indices of significant change in any of the caregiver questionnaire scores were set so that each was approximately $\geq 10 \%$ of the total possible score (ADL $\geq 2$ points, IADL $\geq 3$ points, modified $\mathrm{CDR} \geq 3$ points, $\mathrm{FBI} \geq 3$ points). Indices of bladder health change were set at $\geq 4$ urinary accidents per week to compare before and after the UUM washout period. In addition, RCI scores were used to determine if individual subjects showed significant changes in each of their RBANS index scores after the UUM washout period. Because of the small number of subjects in this UUM study, the computed RCI ranges from another much larger recent study were used to evaluate the reliable change in test scores. In this larger study, 20 control subjects and 20 PD patients were assessed after a clinical intervention using RCIs to determine if their RBANS scores changed significantly [19]. These two study groups (PD and UUM) were nearly identical in the mean age and were from the same locality in Oklahoma, with continual medical supervision. Thus, the RCIs from the larger PD group were used to assess changes in RBANS test scores from the 10 subjects in the UUM study reported here.

Over a 2-year period, we were able to recruit 10 subjects (age range 67-85 years), 5 patients with amnestic MCI, 1 patient with non-amnestic MCI (putative early frontotemporal dementia), and 4 cognitively healthy age-matched subjects. Numbers of subjects recruited were low because many physicians had stopped prescribing oxybutynin, except when treating severe bladder spasms, due to prior complaints from patients of cognitive problems after ingesting that medication.

\section{Statistical Methods}

Descriptive statistics used for analyses included each variable's distribution to determine if it was parametric. Because of the small number of subjects, some variables were not parametric. Therefore, non-parametric analyses were computed using JMP Statistical Program for Professionals (SAS 2000, JMP Statistical Discovery Software; SAS Institute, Inc., Cary, N.C. USA). In addition, raw 'difference' numbers, comparing scores 'on UUM' and 'off UUM', were computed for the two cognitive tests and the four behavioral questionnaire scores; variables showing these differences were computed so that positive numbers showed improvement in function while negative numbers showed a decline in function after the UUM washout period. A basic RCI score was computed using the formula: $\mathrm{RCI}=\mathrm{Y}-\mathrm{Y}^{1} / \mathrm{SE}$ 
Table 1. Demographic characteristics of subjects

\begin{tabular}{lllll}
\hline Subject No. & $\begin{array}{l}\text { Age } \\
\text { years }\end{array}$ & Gender & Ethnicity & MCI \\
\hline 1 & 60 & M & Caucasian & No \\
2 & 78 & M & Caucasian & No \\
$3^{\text {CIM }}$ & 76 & M & American Indian & Yes \\
4 & 67 & $\mathrm{~F}$ & Caucasian & No \\
$5^{\text {CIM }}$ & 82 & M & Caucasian & Yes \\
$6^{\text {CIM }}$ & 85 & M & Caucasian & Yes \\
7 & 71 & M & Caucasian & Yes \\
8 & 67 & M & Caucasian & Yes \\
9 & 69 & M & Caucasian & Yes \\
10 & 75 & M & Caucasian & No \\
\hline \multicolumn{7}{c}{ CIM $=$ Cholinesterase inhibitor medication use. } \\
\hline \multicolumn{5}{c}{} \\
\hline \multicolumn{5}{c}{} \\
\hline \multicolumn{5}{c}{}
\end{tabular}

of difference score, where $\mathrm{Y}$ is the retest score (second score), $\mathrm{Y}^{1}$ the predicted score (first score), and SE the standard error score [21]. ANOVA and regression analyses were used to assess differences between MCI and healthy elderly subjects, as well as between those MCI subjects using a cholinesterase inhibitor medication versus those using no medication. The rationale for the number of subjects recruited into the study was the power analysis from a previous study showing that a significant effect was found for 9 subjects taking both a cholinesterase inhibitor and a UUM [9]. As expected, the healthy elderly subjects had higher overall cognitive test scores compared to the MCI subjects.

\section{Results}

Table 1 includes demographic data for the 10 subjects. Table 2 shows cognitive test results for each subject expressed as the differences between scores off versus on a UUM (positive numbers indicate improvement and negative numbers indicate a decline in skill). Table 3 shows significant changes in behavioral rating scores (approximately $\geq 10 \%$ of total possible score) for each subject after UUM washout. Table 4 summarizes the test scores that significantly differentiated healthy from MCI subjects, showing proper diagnostic accuracy of the 10 subjects.

Although the mean age of the MCI patients was 6 years older than that of the healthy subjects, that difference was not significant $(p=0.24)$. As expected, the 4 healthy elderly subjects scored higher than the $6 \mathrm{MCI}$ subjects on the MMSE. The initial mean MMSE score for the 6 MCI subjects was 25.8 out of a possible 30 points; the washout MMSE mean was 26.2 points. The mean improvement after UUM washout, however, was not significantly different. The first mean MMSE score for the 4 cognitively healthy subjects was $29 / 30$ points; the second mean MMSE score for the healthy subjects was 29.75/30 points (not statistically different). The initial total RBANS mean score for the 4 healthy subjects was significantly higher than the $6 \mathrm{MCI}$ subjects' mean score (51st vs. 10th percentile; $\mathrm{p}=0.02$ ). The UUM washout mean RBANS scores for healthy versus MCI patients showed a non-significant improvement (61st vs. 20th percentile, respectively; $\mathrm{p}=0.3$ ). However, 9 subjects showed significant improvement in one or more discrete cognitive skills after the washout period. The one patient with non-amnestic MCI (No. 6) showed declines in all cognitive skills after UUM washout (table 2). 
Table 2. Differences between cognitive test scores off versus on a UUM

\begin{tabular}{llllllll}
\hline \multirow{2}{*}{$\begin{array}{l}\text { Subject } \\
\text { No. }\end{array}$} & Total & Total & \multicolumn{2}{l}{ RBANS } \\
\cline { 5 - 8 } & MMSE & RBANS & immediate memory & visuospatial & language & attention & delayed memory \\
& 23 & $-13.3,+9.1$ & $-16.6,+18.2$ & $-35.4,+19.6$ & $-9.0,+7.4$ & $-22.4,+16.8$ & $-19.3,+23.0$ \\
\hline $1^{\mathrm{H}}$ & 0 & 2 & -5 & -11 & $61^{*}$ & -4 & -10 \\
$2^{\mathrm{H}}$ & 0 & $20^{*}$ & 0 & $34^{*}$ & -1 & $23^{*}$ & 3 \\
3 & -2 & 2 & 15 & 0 & 0 & 0 & $24^{*}$ \\
$4^{\mathrm{H}}$ & 1 & 5 & 16 & -13 & 0 & $22^{*}$ & 20 \\
5 & 1 & $37^{*}$ & 8 & -4 & $65^{*}$ & 11 & $35^{*}$ \\
6 & $-3^{*}$ & $-15^{*}$ & -11 & -19 & -2 & -14 & -4 \\
7 & 2 & $13^{*}$ & 15 & $31^{*}$ & $44^{*}$ & 0 & 0 \\
8 & 1 & $14^{*}$ & $30^{*}$ & 11 & 0 & -2 & $48^{*}$ \\
9 & $4.5^{*}$ & 2.8 & 0.3 & 8 & $54^{*}$ & 15 & 0 \\
$10^{\mathrm{H}}$ & 0 & $11^{*}$ & 0 & 0 & $23^{*}$ & 15 & 4 \\
\hline
\end{tabular}

RBANS: RCI + practice effect score range for significant change. Significant differences were set at $\geq 3$ pts for MMSE (10\% change). The RCI + practice effect score ranges are equivalent to a Z score of 1.645 or $90 \%$ and were derived from a large study of subjects [19] similar to the small case study group described here. Note that each subject showed a different profile of total and subtest score changes.

$\mathrm{H}=$ Healthy elderly subjects. ${ }^{*}$ Significant difference in scores.

Table 3. Differences in behavioral indices after UUM washout

\begin{tabular}{|c|c|c|c|c|c|c|}
\hline $\begin{array}{l}\text { Subject } \\
\text { No. }\end{array}$ & $\begin{array}{l}\text { FBI } \\
\text { difference } \\
\text { (0-24 points) }\end{array}$ & $\begin{array}{l}\text { M-CDR } \\
\text { difference } \\
\text { (0-28 points) }\end{array}$ & $\begin{array}{l}\text { ADL } \\
\text { difference } \\
\text { (0-18 points) }\end{array}$ & $\begin{array}{l}\text { IADL } \\
\text { difference } \\
\text { (0-27 points) }\end{array}$ & $\begin{array}{l}\text { Number of } \\
\text { urinary } \\
\text { accidents/ } \\
\text { week difference }\end{array}$ & UUM \\
\hline $1^{\mathrm{H}}$ & 2 & 1 & $2^{*}$ & 1 & 2 & oxybutynin \\
\hline $2^{\mathrm{H}}$ & -2 & -1 & $-2^{*}$ & 1 & 2 & oxybutynin \\
\hline 3 & 1 & $7^{*}$ & 0 & 1 & 1 & oxybutynin \\
\hline $4^{\mathrm{H}}$ & 0 & 0 & 0 & 0 & 0 & tolterodine \\
\hline 5 & $3^{*}$ & $12^{*}$ & $3^{*}$ & $4^{*}$ & 2 & oxybutynin \\
\hline 6 & $-5^{*}$ & -1 & $-5^{*}$ & -1 & 0 & oxybutynin \\
\hline 7 & 1 & $5^{*}$ & 0 & 1 & 0 & oxybutynin \\
\hline 8 & -1 & 0 & 0 & 1 & 1 & oxybutynin \\
\hline 9 & $8^{*}$ & $4.5^{*}$ & 0 & $5^{*}$ & 3 & oxybutynin \\
\hline $10^{\mathrm{H}}$ & $5^{*}$ & 0 & 0 & 0 & 1 & oxybutynin \\
\hline
\end{tabular}

Scores were taken from behavioral questionnaires and urinary accident diaries filled out by the family member living with the subject. Four standard questionnaires have been designed to indicate more pathology by higher scores (FBI, CDR, ADL, IADL), so difference scores for these instruments were computed as the score on - score off a UUM. Positive numbers show less pathology; negative numbers show more pathology. Figures for significant changes were established before the study began and were set to equal approximately $\geq 10 \%$ of the total possible scores for each questionnaire (FBI $\geq 3$; modified CDR $\geq 3$; $\mathrm{ADL} \geq 2$; IADL $\geq 3$; urinary accidents per week $\geq 4$ ).

Note that no significant differences in urinary accident rates were noted for any subject. One male MCI patient on oxybutynin (No. 8) showed non-significant changes, and the healthy female on tolterodine showed no behavioral differences at all (No. 4).

$\mathrm{H}=$ Healthy elderly subjects; M-CDR = modified CDR. ${ }^{*}$ Significant difference in scores. 
Table 4. Scores differentiating cognitively normal from MCI subjects

Monnot et al.: Urinary Urgency Medications May Compromise Discrete rather than Global Cognitive Skills

\begin{tabular}{|c|c|c|c|c|}
\hline Statistical analysis & Probability & $\mathrm{t}$ test & $\begin{array}{l}\text { Adjusted } \\
\mathrm{R}^{2}\end{array}$ & F ratio \\
\hline ANOVA of 1st MMSE & $\mathrm{p}=0.03$ & 2.6 & 0.39 & 6.7 \\
\hline ANOVA of 2nd MMSE & $\mathrm{p}=0.04$ & 2.4 & 0.34 & 5.6 \\
\hline ANOVA of 1st RBANS & \multirow[t]{2}{*}{$\mathrm{p}=0.02$} & & & \\
\hline Total percentile rating & & 2.7 & 0.41 & 7.3 \\
\hline ANOVA of 2nd RBANS & \multirow[t]{2}{*}{$\mathrm{p}=0.03$} & & & \\
\hline Total percentile rating & & 2.5 & 0.37 & 6.4 \\
\hline ANOVA of 1st RBANS subtest & $p=0.03$ & & & \\
\hline Immediate memory & & 2.7 & 0.41 & 7.2 \\
\hline ANOVA of 1st RBANS subtest & $\mathrm{p}=0.04$ & & & \\
\hline Delayed memory & & 2.4 & 0.35 & 5.8 \\
\hline ANOVA of 2 nd RBANS subtest & $\mathrm{p}=0.02$ & & & \\
\hline Visuospatial construct & & 3.0 & 0.47 & 8.8 \\
\hline ANOVA of 2nd RBANS subtest & $\mathrm{p}=0.04$ & & & \\
\hline Attention/concentration & & 2.5 & 0.36 & 6.1 \\
\hline
\end{tabular}

Probability alpha was set at 0.05 for all $t$ tests and ANOVAs. Figures are rounded. Analyses showed that all other cognitive tests and behavioral questionnaires did not significantly differentiate between subjects with MCI and normal cognition for age and education level.

There were no significant relationships found between subject age and the 'difference scores' (on vs. off UUM) for ADL, MMSE, total-RBANS, FBI, and IADL assessments ( $\mathrm{p}=$ 0.063 to $\mathrm{p}=0.81$, respectively). No significant difference was found between age and subject diagnosis (MCI vs. cognitively healthy; $\mathrm{p}=0.24$ ). However, large differences in RBANS subtest scores were found for individual subjects after the washout period; each subject showed different patterns of changes depending upon the skill being tested (table 2). In addition, no significant differences in the rates of urinary accidents were found for any subject when the baseline week 'on' their UUM was compared to the last week of the washout period; accident rates were essentially the same whether the patient was taking a UUM or not (table 3). Though the MCI subjects were rated as having slightly more behavior problems than the healthy subjects, no significant differences were found between the two groups on any rating questionnaire or on the cognitive problems diary counts $(\mathrm{p}=0.08$ to $\mathrm{p}=0.65)$, either before or after the UUM washout. The only significant relationship between the 'urinary accident diary' counts and Bladder Health Questionnaire scores was for responses during the last week of the UUM washout for the 4 healthy subjects $\left(\mathrm{p}=0.05, \mathrm{r}=0.94, \mathrm{r}^{2}=0.88\right)$; no significant relationships between these two urinary behavior measures were found for the $6 \mathrm{MCI}$ patients at any time. No significant relationships were found for cognitive or behavior rating scores with MCI subjects' use of a cholinesterase inhibitor medication (see table 1).

\section{Discussion}

Mixed results from prior studies of cognitive status associated with the use of oxybutynin (or tolterodine) appear to be related to methodological differences. In the study described here, initial and subsequent test scores showed that the healthy subjects were more competent than the MCI subjects. When repeated measures of total test scores were calculated to show any difference after the UUM washout period, the overall results often failed to show significant differences, as reported in some prior publications $[12,16]$. 
Monnot et al.: Urinary Urgency Medications May Compromise Discrete rather than Global Cognitive Skills

However, the subtests of the RBANS showed quite clearly that each of the 10 subjects in this study displayed different patterns of cognitive improvement, except for the patient with non-amnestic MCI who showed consistent declines in the cognitive and behavioral measures (see table 2). In other words, some subjects showed a difference in language ability and in attention/concentration, while others showed a difference in visuospatial skills and in immediate memory, etc. Nine subjects showed one or more significant improvements in cognitive subtest scores, with 5 individuals showing significant changes in language; 8 subjects showed one or more significantly improved behavior rating scores (see table 3). Subject No. 6 was the only patient diagnosed with non-amnestic MCI; all scores declined after the washout period, with total scores for MMSE and RBANS tests showing significant changes. The other 5 patients diagnosed with amnestic MCI showed significant improvement on one or more subtest scores, but with each subject showing a different pattern of changes. The 4 healthy elderly subjects also showed significant subtest score improvements after the UUM washout period. Six of the 10 subjects showed significant changes in their total RBANS score, and 2 of these 10 subjects showed significant changes in their MMSE total scores after UUM washout.

Thus, total test scores from multidimensional instruments, such as the RBANS, may not be sensitive enough to reveal subtle but bothersome cognitive problems that arise with the use of a UUM or other medications. Although we do not know why UUMs affect discrete cognitive skills, one possibility is that these medications impact a subject's 'weakest' cognitive skill(s), rather than all cognitive skills.

None of the subjects reported any significant differences in their urinary habits/symptoms by the end of this study. Apparently, in this small sample, a 4-week washout (withdrawal) of oxybutynin and tolterodine did not appreciably affect the subjects' urinary accident rate compared to the rate established at the beginning of this study. There was no association between scores on the Bladder Health Questionnaire and the urinary accident diary counts for the $6 \mathrm{MCI}$ patients $(\mathrm{p}=0.42)$, though these two scores were significantly positively associated for the 4 healthy subjects $(\mathrm{p}=0.5, \mathrm{r}=0.95)$. Though still competent, reduced memory skills for the MCI subjects may have affected their responses on self-report inventories. Age and diagnosis of subjects were not significantly associated with the difference scores (the changes in cognition and/or behavior noted after the UUM washout period). Apparently, the two studied medications alone were responsible for the changes in cognition and behavior reported by family members after the UUM washout period, rather than each subject's cognitive diagnosis (MCI vs. healthy controls).

It should be noted that the one patient diagnosed as non-amnestic MCI lost points on all tests when the anticholinergic UUM was discontinued. In our 15 years of focused dementia care, we have found that some patients with early frontotemporal dementia may show increased neuropsychiatric symptoms, such as agitation, when given cholinesterase inhibitor medication as opposed to amnestic-MCI and AD patients.

Though methodology was the focus of this study, it should be emphasized that some older UUMs appear to be associated with cognitive problems in both healthy elderly subjects and elderly MCI patients. Because no change in urinary habits after the UUM washout was found in this small case study, the risk of reducing discrete cognitive skills makes the use of these two UUMs questionable for urinary urgency. Other medical management may be more beneficial for such patients.

Our results should be considered when designing methodology for future studies because they show that each subject has a different profile of discreet cognitive and behavioral responses to a medication. Therefore, details of methodology used to assess subjects are vital to the process of arriving at reliable conclusions. Evaluating the pattern of changes for several individual skills, rather than relying only on a total test score or a group mean number, appears to yield more useful and comprehensive data about cognitive/behavioral changes 
after medical intervention or in drug trials. These methods should be considered in the future to plan more efficacious trials that can avoid the inconsistencies reported previously in this area of medical research.

\section{Acknowledgement}

The authors wish to thank James G. Scott, PhD, Department of Psychiatry and Behavioral Sciences, University at Oklahoma Health Sciences Center, for his review of the manuscript and for his valuable suggestions for improvements.

\section{References}

1 Mesulam M, Shaw P, Mash D, Weintraub S: Cholinergic nucleus basalis tauopathy emerges early in the aging-MCI-AD continuum. Ann Neurol 2004;55:815-828.

-2 Agnoli A, Martucci N, Manna V, Conti L, Fioravanti M: Effects of cholinergic and anticholinergic drugs on short-term memory in Alzheimer's dementia: a neuropsychological and computerized electroencephalographic study. Clin Neuropharmacol 1983;6:311-323.

-3 Katz I, Sands L, Bilker W, DiFilippo S, Boyce A, D’Angelo K: Identification of medications that cause cognitive impairment in older people: the case of oxybutynin chloride. J Am Geriatr Soc 1998;46: $8-13$.

-4 Banerjee S, Routledge PA, Pugh S, Smith PM: Poisoning with oxybutynin. Hum Exp Toxicol 1991; 10:225-226.

5 Pietzko A, Dimpfel W, Schwantes U, Topfmeier P: Influence of trospium chloride and oxybutynin on quantitative EEG in healthy volunteers. Eur J Clin Pharmacol 1998;47:337-343.

-6 Todorova A, Vonderheid-Guth B, Dimpfel W: Effects of tolterodine, trospium chloride, and oxybutynin on the central nervous system. J Clin Pharmacol 2001;41:636-644.

-7 Diefenbach K, Arnold G, Wollny A, Schwantes U, Haselmann J, Roots I: Effects on sleep of anticholinergics used for overactive bladder treatment in healthy volunteers aged $>$ or $=50$ years. BJU Int 2005;95:346-349.

-8 Chew ML, Mulsant BH, Pollock BG: Serum anticholinergic activity and cognition in patients with moderate-to-severe dementia. Am J Geriatr Psychiatry 2005;13:535-538.

-9 Jewart R, Green J, Lu Ching-ju, Cellar J, Tune LE: Cognitive, behavioral, and physiological changes in Alzheimer disease patients as a function of incontinence medications. Am J Geriatr Psychiatry 2005; 13:324-328.

-10 Chew ML, Mulsant BH, Pollock BG, Lehman ME, Greenspan A, Maumoud RA, Kirshner, MA, Sorisio DA, Bies RR, Gharabawi G: Anticholinergic activity of 107 medications commonly used by older adults. J Am Geriatr Soc 2008;56:1333-1341.

-11 Robinson M, Rowett D, Leverton A, Mabbott V: Changes in utilization of anticholinergic drugs after initiation of cholinesterase inhibitors. Pharmacoepidemiol Drug Saf 2009;18:659-664.

-12 Brown JS, Vittinghoff E, Wyman JF, Stone KL, Nevitt MC, Ensrud KE, Grady D: Urinary incontinence: does it increase risk for falls and fractures? Study of Osteoporotic Fractures Research Group. J AM Geriatr Soc 2000;48:721-725.

-13 Lackner TE, Wyman JF, McCarthy TC, Monigold M, Davey C: Randomized, placebo-controlled trial of the cognitive effect, safety, and tolerability of oral extended-release oxybutynin in cognitively impaired nursing home residents with urge urinary incontinence. J Am Geriatr Soc 2008;56:862870.

-14 Sink KM, Thomas J 3rd, Xu H, Craig B, Kritchevsky S, Sands LP: Dual use of bladder anticholinergics and cholinesterase inhibitors: long-term functional and cognitive outcomes. J Am Geriatr Soc 2008; 56:847-853.

15 Giramonti KM, Kogan BA, Halpern LF: The effects of anticholinergic drugs on attention span and short-term memory skills in children. Neurourol Urodyn 2008;27:315-318. 
Monnot et al.: Urinary Urgency Medications May Compromise Discrete rather than Global Cognitive Skills

-16 Wesnes KA, Edgar C, Tretter RN, Bolodeoku J: Exploratory pilot study assessing the risk of cognitive impairment or sedation in the elderly following single doses of solifenacin $10 \mathrm{mg}$. Expert Opin Drug Saf 2009;8:615-626.

$\checkmark 17$ Kay GG, Eginger U: Preserving cognitive function for patients with overactive bladder: evidence for a differential effect with darifenacin. Int J Clin Pract 2008;62:1641-1642.

18 Duff K, Patton DE, Schoenberg MR, Mold J, Scott JG, Adams RL: Intersubtest discrepancies on the RBANS: results from the OKLAHOMA study. Appl Neuropsychol 2011;18:79-85.

19 Schoenberg MR, Rinehardt E, Duff K, Mattingly M, Bharucha KG, Scott JG: Assessing reliable change using the repeatable battery for the assessment of neuropsychological status (RBANS) for patients with Parkinson's disease undergoing deep brain stimulation (DBS) surgery. Clin Neuropsychol 2012;26:255-270.

-20 Maassen GH, Bossema E, Brand N: Reliable change and practice effects: outcomes of various indices compared. J Clin Exp Neuropsychol 2009;31:339-352.

-21 Hinton-Bayre AD: Deriving reliable change statistics from test-retest normative data: comparison of models and mathematical expressions. Arch Clin Neuropsychol 2010;25:244-256.

-22 Mitrushima M, Drebing C, Satz P, Van Gorp W, Chervinsky A, Uchiyama C: WAIS-R intersubtest scatter in patients with dementia of Alzheimer's type. J Clin Psychol 1994;50:753-758.

23 Randolph C: Repeatable Battery for the Assessment of Neuropsychological Status. Psychological Corporation, Harcourt Assessment Company, 1998.

-24 Ford GR, Haley WE, Thrower SL, West CA, Harrell LE: Utility of Mini-Mental State Exam scores in predicting functional impairment among white and African American dementia patients. J Gerontol A Biol Sci Med Sci 1996;51:M185-M188.

-25 Crawford JR, Garthwaite PH: Percentiles please: case for expressing neuropsychological test scores and accompanying confidence limits as percentile ranks. Clin Neuropsychol 2009;23:193-204.

-26 Monnot M, Brosey M, Ross E: Screening for dementia: family caregiver questionnaires reliably predict dementia. J Am Board Fam Pract 2005;18:240-256. 